\title{
Myocardial bridging in a survivor of sudden cardiac near-death: role of intracoronary Doppler flow measurements and angiography during dobutamine stress in the clinical evaluation
}

\author{
René A Tio, Isabel C Van Gelder, Piet W Boonstra, Harry J G M Crijns
}

\begin{abstract}
Extensive myocardial bridging in the left anterior descending coronary artery was found in a 46 year old survivor of sudden cardiac near-death. Positron emission tomography and dobutamine stress echocardiography revealed ischaemia in the myocardium distal to the bridging. Spasm was excluded as cause of the ischaemia by intracoronary infusion of acetylcholine. Further evaluation of the haemodynamic importance of the bridging using intracoronary Doppler flow velocity measurements revealed an abnormal flow reserve. Dobutamine stress during coronary angiography caused increased mechanical compression during diastole. This was accompanied by multiple premature ventricular contractions. After a debridging operation the flow velocity reserve was normal. The abnormalities found during dobutamine stress had disappeared. Unexpectedly, a spasm was inducible. This may have been due to local oedema or scar formation after the operation. For the evaluation of the haemodynamic importance of myocardial bridging, intracoronary Doppler flow velocity measurements
\end{abstract}

and angiography during dobutamine stress may be helpful in clinical decision making.

(Heart 1997;77:280-282)

Keywords: dobutamine stress; coronary flow reserve; 윽 myocardial bridging; sudden cardiac death

Sudden cardiac death in young and middle $\stackrel{\text { Oิ }}{工}$ aged subjects may be caused by (congenital) $\vec{\bullet}$ abnormalities of the coronary arteries. In this report a case of extreme myocardial bridging was found as the precipitating factor for ven-o tricular arrhythmia in a survivor of sudden cardiac near-death.

\section{Case}

A 46 year old man collapsed during lunch in a factory canteen. Cardiopulmonary resuscitation was started immediately. On the arrival of the ambulance ventricular fibrillation was pre- $\overparen{\Phi}$ sent. After defibrillation, sinus rhythm and adequate circulation were established. In the hospital the heart rate was 50 beats $/ \mathrm{min}$, blood pressure $130 / 70 \mathrm{~mm} \mathrm{Hg}$, and the patient $ᄋ$ respired spontaneously. No neurological
Department of Cardiology, Thoraxcenter, University Hospital Groningen, Groningen, The Netherlands R A Tio I C van Gelder H J G M Crijns

Department of Cardiopulmonary Surgery,

Thoraxcenter, University Hospital Groningen, Groningen, The Netherlands P W Boonstra Correspondence to: Dr R A Tio, Department of Cardiology, Thoraxcenter, University Hospital Groningen, PO Box 30.001, $9700 \mathrm{RB}$ Groningen, The Netherlands.

Accepted for publication 6 September 1996

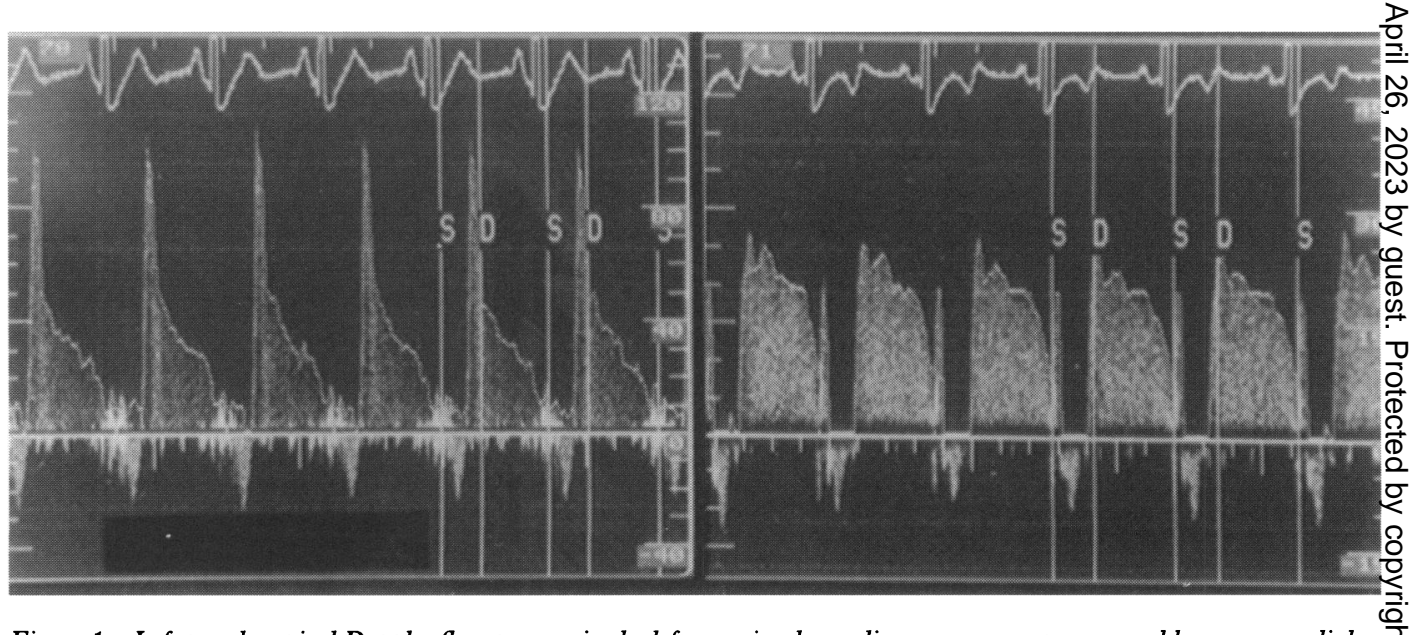

Figure 1 Left panel: typical Doppler flow pattern in the left anterior descending coronary artery caused by a myocardial $\stackrel{2}{\supset}$ bridge. Marked retrograde flow during systole is present. During early diastole a high peak flow is seen, followed by a gradual normalisation of the flow. Right panel: normalisation of the Doppler flow pattern after the debridging operation. There is still some retrograde flow during systole, but to a lesser extent. During the diastole the early peak has disappeared. The number in the upper left hand corner of each panel indicates the heart rate. The scale at the right of each panel indicates the average peak velocity. 
Figure 2 Diastolic $(A)$ and systolic (B) angiographic frames from the left superior oblique view before dobutamine infusion; diastolic (C) and systolic (D) frames during intravenous dobutamine (40 $\mu \mathrm{g} / \mathrm{kg} / \mathrm{min})$ stress; and the same diastolic $(E)$ and systolic frames $(F)$ after the debridging operation.
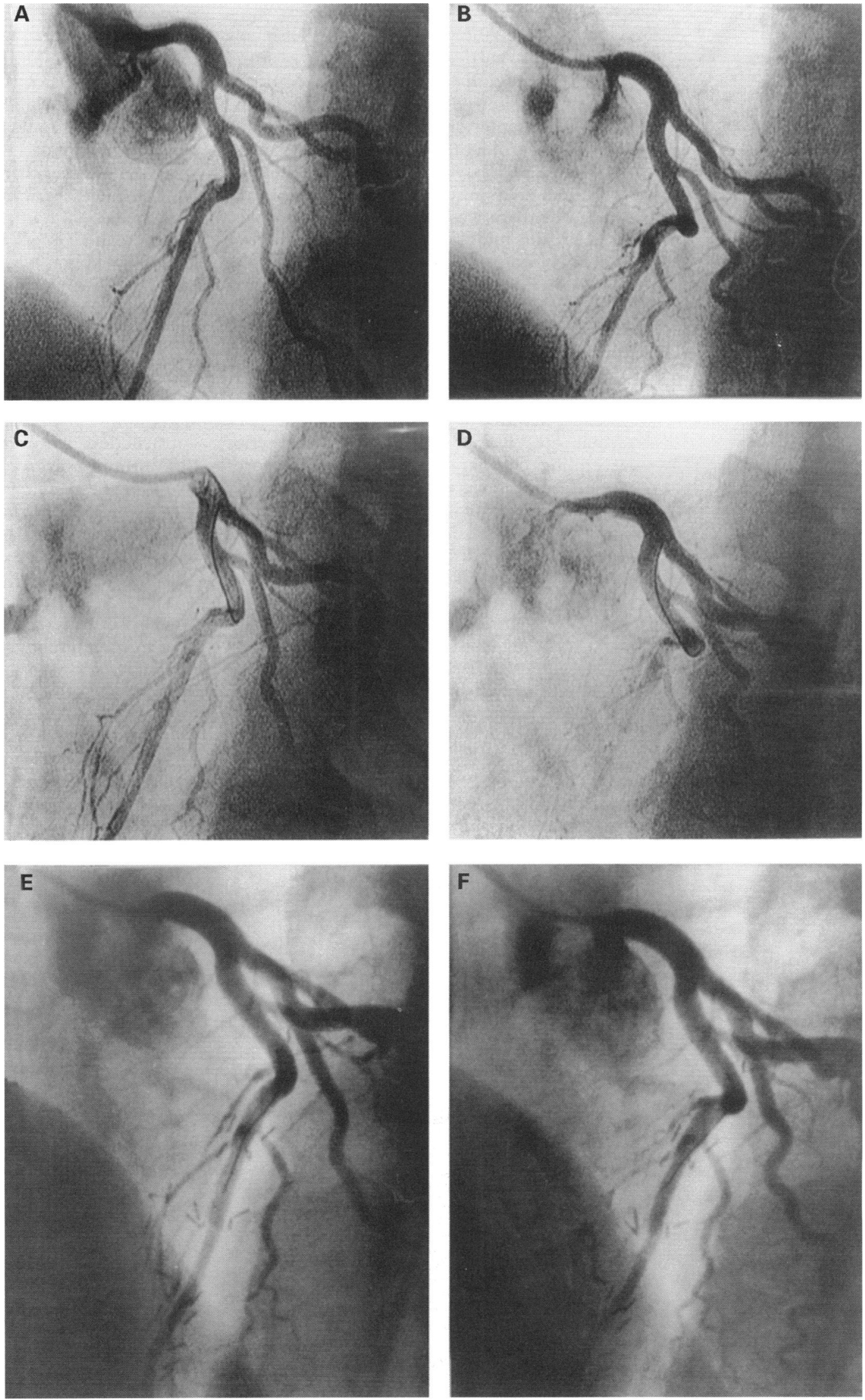

deficit was present. Neither the electrocardiogram nor the laboratory measurements indicated myocardial infarction.

The past medical history was unremarkable except for the electrocardiographic and echocardiographic diagnosis of left ventricular hypertrophy four years previously in another hospital. The exercise test and 24 hour electrocardiography were normal at that time.

During hospital admission, an analysis of the cause of the patient's cardiovascular collapse was undertaken. On the electrocardiogram, negative $T$ waves were found in the precordial and inferior leads. An echocardiogram 
revealed normal wall motion in all segments. Magnetic resonance imaging did not show signs of right ventricular dysplasia. A positron emission tomogram $\left({ }^{13} \mathrm{~N}\right.$-ammonia; dypiridamole stress) showed ischaemia in the apical part of the anterior wall, and dobutamine stress echocardiography showed hypokinesia in the same region. A coronary angiogram was performed. No atherosclerotic lesions were found, but there was an extensive myocardial bridge in the middle part of the left anterior descending coronary artery. Using a Doppler flow wire (Flomap; Cardiometrics, Mountain View, California, USA) a remarkable ("spike and dome") flow pattern was found in the left anterior descending coronary artery (fig 1, left panel). An abnormal coronary flow velocity reserve (intracoronary papaverine $10 \mathrm{mg}$, Flomap software for peak detection; ratio 1.3; normal >2) was found in the left anterior descending coronary artery. With intracoronary infusion of acetylcholine (up to $1 \mu \mathrm{mol} / \mathrm{l}$ ) no spasm was inducible. Finally, after increasing doses of dobutamine (up to $40 \mu \mathrm{g} / \mathrm{kg} / \mathrm{min}$ ) the left anterior descending coronary artery was compressed entirely during the systole. This was accompanied by frequent uniform premature ventricular contractions, presumably originating from the septal region.

The patient underwent a debridging operation using a heart-lung machine and cardioplegic arrest. A myocardial bridge $7 \mathrm{~cm}$ long and at most $5 \mathrm{~mm}$ thick was cleaved. After the operation no signs of ischaemia were found (dobutamine stress echo and positron emission tomogram). No bridging was observed at repeated angiography (fig 2; three weeks after operation). The flow reserve was 3.0 and the Doppler flow pattern was normal (fig 1B). During dobutamine infusion the left anterior descending coronary artery was no longer compressed and no premature ventricular contractions were present. However, a spasm was inducible with acetylcholine (at a dose of $1 \mu \mathrm{mol} / \mathrm{l}$ ), but with no symptoms or rhythm disturbances. The patient left the hospital and was given a calcium antagonist for routine use. $\mathrm{He}$ is doing well six months after discharge.

\section{Discussion}

The prevalence of myocardial bridges in anatomical studies varies from $5 \cdot 4 \%$ to $85.7 \%$, depending on definition and method used $\left(\mathrm{see}^{2}\right.$ for review). During angiography systolic narrowing of a coronary artery segment is seen in $0.5 \%$ to $4.5 \%$, the most frequent site being the middle segment of the left anterior descending coronary artery.

Although most of the myocardial bridges are thought to be innocent, ${ }^{2}$ in some cases they may cause angina pectoris, ${ }^{3}$ myocardial infarction, ${ }^{3-5}$ life threatening ventricular arrhyth- mias, or even death. ${ }^{67}$ The underlying mechanism of these arrhythmias may be ischaemic, related to flow limitation by the myocardial bridge. Because myocardial perfusion takes place mainly during the diastole, the systolic narrowing is thought not to be capable of causing ischaemia, which may therefore be caused by other mechanisms: for example, because of the continuous mechanical stress endothelial cell damage may occur. This predisposes the vessel segment to vasospasm ${ }^{89} \stackrel{\text { की }}{\rightarrow}$ and may explain why myocardial bridges can cause ischaemia. Another mechanism might $\frac{\bar{\sigma}}{\bar{s}}$ be premature atherosclerosis at the site of the myocardial bridge.

In our patient no such mechanism was found. However, the coronary flow reserve $\overrightarrow{0}$ distal to the bridge was abnormally low, $\vec{A}$ suggesting a haemodynamically important $\omega_{\text {D }}$ "obstruction" with corresponding abnormalities during positron emission tomography and stress echocardiography. These findings sug- iे gest that extreme bridges, such as in this case, $i$ may cause ischaemia in the absence of coro- $\mathscr{O}$ nary vasospasm.

The choice of cardiac surgery in this patient was based on the length of the bridging. In $\vec{z}$ other cases, angioplasty or even stenting may

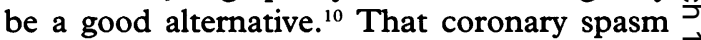
was inducible after the operation may have $\notin$ been the result of an inflammatory reaction in. the vessel wall and postoperative scar formation. The cause of ischaemia in patients with myocardial bridging is probably multifactorial. In individual patients all potential mechanisms have to be considered. Thus, as well as stress $\stackrel{\perp}{\perp}$ angiography and spasm provocation, the mea- $\overrightarrow{\vec{O}}$ surement of the coronary flow reserve may be 3 considered useful in the evaluation of the clinical significance of a myocardial bridge.

1 Flynn MS, Kern MJ, Aguirre FV, Bach RG, Caraciolo EA, Donohue TJ. Intramyocardial muscle bridging of the coronary artery - an examination of a diastolic "spike and dome" pattern of coronary flow velocity. Cath Cardiovasc Diagn 1994;32:36-9.

2 Angelini P, Trivellato M, Donis J, Leachman RD. Myocardial bridges: a review. Prog Cardiovasc Dis 1983; 윽

3 Faruqui AMA, Maloy WC, Felner JM, Schlant RC, Logan I WD, Symbas P. Symptomatic myocardial bridging of coronary artery. Am f Cardiol 1978;41:1305-10.

4 Feldman AM, Baughman. Myocardial infarction associated $\mathrm{N}$ with a myocardial bridge. Am Heart f 1986;111:784-7.

5 Brito FS, Brito FS. Disappearance of systolic narrowing of a $N$ mural coronary artery after myocardial infarction. $\mathrm{Am}$ mural coronary artery after myocardial infarction. Am N Morales AR, Romanelli R, Boucek RJ. The mural left ante- 0 rior descending coronary artery, strenuous exercise and $<$ sudden death. Circulation 1980;62:230-7.

7 Kracoff OH, Ovsyshscher I, Gueron M. Malignant course of a benign anomaly: myocardial bridging. Chest 1987; $\mathbb{E}$ 92:1113-5.

8 Grover M, Mancini GBJ. Myocardial bridge associated $T$ with pacing induced coronary spasm. Am Heart $\mathcal{F} 1984$; 108:1540-3.

9 Ciampricotti R, El Gamal M. Vasospastic coronary occlusion associated with a myocardial bridge. Cath Cardiovasc

10 Stables RH, Knight CJ, Sigwart U. Coronary stenting in the management of myocardial ischaemia caused by muscle bridging. Br Heart $\mathcal{F} 1995 ; 74: 90-2$. 